\title{
Differential Cellular and Subcellular Localization of AMPA Receptor- Binding Protein and Glutamate Receptor-Interacting Protein
}

\author{
Alain Burette, ${ }^{1}$ Latika Khatri, ${ }^{2}$ Michael Wyszynski, ${ }^{3}$ Morgan Sheng, ${ }^{3}$ Edward B. Ziff, ${ }^{2}$ and \\ Richard J. Weinberg ${ }^{1}$ \\ ${ }^{1}$ Department of Cell Biology and Anatomy, University of North Carolina, Chapel Hill, North Carolina 27599, ${ }^{2 H o w a r d}$ \\ Hughes Medical Institute and Department of Biochemistry, New York University Medical School, New York, New York \\ 10016, and ${ }^{3 H o w a r d}$ Hughes Medical Institute and Department of Neurobiology, Massachusetts General Hospital and \\ Harvard Medical School, Boston, Massachusetts 02114
}

Excitatory synaptic currents in the mammalian brain are typically mediated by the neurotransmitter glutamate, acting at AMPA receptors. We used immunocytochemistry to investigate the distribution of AMPA receptor-binding protein (ABP) in the cerebral neocortex. ABP was most prominent in pyramidal neurons, although it was also present (at lower levels) in interneurons. ABP and its putative binding partners, the GluR2/3 subunits of the AMPA receptor, exhibited prominent cellular colocalization. Under appropriate processing conditions, colocalization could also be documented in puncta, many of which could be recognized as dendritic spines. However, a sizable minority of GluR2/3-positive puncta were immunonegative for ABP. Because glutamate receptor-interacting protein (GRIP) may also anchor GluR2, we studied the relative distribution of ABP and GRIP. There was extensive colocalization of these two

Ionotropic glutamate receptors concentrate at the plasma membrane overlying the excitatory postsynaptic density (PSD) (for review, see Ottersen and Landsend, 1997; Conti and Weinberg, 1999; Nusser, 2000). Investigation of the molecular basis for this phenomenon led to the discovery that PSD-95, a novel protein concentrated at the PSD, binds to the NMDA receptor (for review, see Kennedy, 1997; O'Brien et al., 1998). PSD-95 is one of a family of proteins expressing PDZ domains, modular sites for protein-protein interaction shared by PSD-95, the Discs-large protein in Drosophila, and ZO-1, a protein found at the epithelial zonula occludens (for review, see Ponting et al., 1997; Sheng and Wyszynski, 1997). The second PDZ domain of PSD-95 binds to the C-terminal motif of the NR2 subunit (Kornau et al., 1995; Niethammer et al., 1996). Besides helping to anchor NMDA receptors, PSD-95 may regulate second-messenger pathways by bringing the receptor into proximity with downstream signaling molecules such as the scaffolding molecule InaD, the PDZ domains of which are crucial organizers of the Drosophila phototransduction cascade (for review, see Ziff, 1997; Craven and Bredt, 1998; Migaud et al., 1998; Scott and Zuker, 1998).

\footnotetext{
Received July 31, 2000; revised Oct. 25, 2000; accepted Oct. 30, 2000.

This work was supported by National Institutes of Health Grants NS35527 (R.J.W.), AG13620 (E.B.Z.), and NS35050 (M.S.). E.B.Z. is an investigator and M.S. is an assistant investigator of the Howard Hughes Medical Institute. We thank R. J. Wenthold for antibodies against GluR2/3.

Correspondence should be addressed to Richard Weinberg, Department of Cell Biology and Anatomy, CB 7090, University of North Carolina, Chapel Hill, NC 27599. E-mail: rjw@med.unc.edu.

Copyright (C) 2001 Society for Neuroscience $\quad 0270-6474 / 01 / 210495-09 \$ 15.00 / 0$
}

antigens at the cellular level, although GRIP, unlike ABP, was strongest in nonpyramidal neurons. Different parts of a single dendrite could stain selectively for ABP or GRIP. To further characterize this heterogeneity, we investigated punctate staining of neuropil using synaptophysin and the membrane tracer DiA to identify probable synapses. Some puncta were comparably positive for both $\mathrm{ABP}$ and GRIP, but the majority were strongly positive for one antigen and only weakly positive or immunonegative for the other. This heterogeneity could be seen even within adjacent spines of a single dendrite. These data suggest that ABP may act as a scaffold for AMPA receptors either in concert with or independently from GRIP.

Key words: cerebral cortex; pyramidal neurons; PDZ; scaffolding proteins; GluR2/3; AMPA receptors; ABP; GRIP

Synapses in cerebral cortex and hippocampus commonly coexpress NMDA and AMPA receptors (Bekkers and Stevens, 1989; Kharazia et al., 1996; He et al., 1998; Takumi et al., 1999; Nusser, 2000). However, NMDA and AMPA receptors lie at different loci within the synaptic active zone (Kharazia and Weinberg, 1997; Somogyi et al., 1998). Yeast two-hybrid screens have identified glutamate receptor-interacting protein (GRIP), a protein containing seven PDZ domains that can bind to the AMPA subunit GluR2 in vitro (Dong et al., 1999), and more recently, AMPA receptorbinding protein (ABP), and its splice variant, ABP-L, sometimes termed GRIP-2, a homologous protein coded on a different gene (Srivastava et al., 1998; Bruckner et al., 1999; Dong et al., 1999; Wyszynski et al., 1999). Biochemical evidence indicates an association between several PDZ domains of ABP and the $\mathrm{C}$ termini of the GluR2/3 subunits (Dong et al., 1999; Wyszynski et al., 1999), but there is no structural evidence that ABP is actually associated with synaptic glutamate receptors in intact brain. Moreover, it remains unclear what the functional significance of two related AMPA-binding proteins may be. Do they function in concert to help organize the AMPA receptor signaling pathway, or do they act independently? If the two proteins act cooperatively, both must be at the same synapse. However, GRIP in cerebral neocortex concentrates in specific classes of inhibitory local circuit neurons, whereas the limited evidence available suggests a more widespread distribution for ABP (Srivastava et al., 1998; Burette et al., 1999; Dong et al., 1999).

These considerations motivated us to ask whether ABP colocalizes with GluR2/3, and if so, whether ABP and GRIP colocalize. 
We provide direct evidence for synaptic colocalization of ABP with GluR2/3 in the adult cerebral cortex and document an unexpectedly complex pattern of colocalization between ABP and GRIP.

\section{MATERIALS AND METHODS}

Antiserum. ABP antibody was generated by immunizing rabbits with a peptide corresponding to amino acids $759-774$, lying within its unique $\mathrm{C}$-terminal L2 region. An extra C-terminal Cys was included to permit glutaraldehyde conjugation to keyhole limpet hemocyanin (KLH) (Srivastava et al., 1998). This antibody recognizes both ABP and ABP-L/ GRIP-2. Two anti-GRIP antibodies were used. Antibody 1756/B.O. was raised in rabbits against a hexahistidine-tagged fusion protein incorporating GRIP amino acids $664-1112$, including PDZ6 and extending to the $\mathrm{C}$ terminus of the protein (for characterization, see Wyszynski et al., 1998, 1999). Because of concerns regarding possible cross-reaction, we also used a second affinity-purified antibody that was generated by immunizing rabbits with a peptide corresponding to GRIP amino acids 1084-1100 (SDWSEQNSAFFQQPSHG), conjugated to KLH through a cysteine added to the $\mathrm{N}$ terminus of the peptide (S. deSouza and $\mathrm{E}$. Z iff, unpublished observations); this region of GRIP is not shared by ABP, making the possibility of cross-reaction unlikely.

Tissue preparation. All procedures related to the care and treatment of animals were in accordance with institutional and National Institutes of Health guidelines. Nine male Sprague Dawley rats (200-350 gm) (Charles River Laboratories, Raleigh NC) were used for this study. Animals were deeply anesthetized with sodium pentobarbital $(60 \mathrm{mg} / \mathrm{kg}$, i.p.). After intracardiac injection of heparin, six rats were perfused through the heart with saline, followed by freshly depolymerized $4 \%$ paraformaldehyde in phosphate buffer (PB; $0.1 \mathrm{M}, \mathrm{pH}$ 7.4). Transverse sections $20-70 \mu \mathrm{m}$ thick were cut on a vibratome and collected in cold PB. To assess whether limited antibody access consequent to aldehyde cross-linking may influence results, we perfused five animals with $0.5 \%$ paraformaldehyde, followed by a saline flush. In additional experiments, we performed partial proteolysis on material fixed with $4 \%$ paraformaldehyde (Watanabe et al., 1998; Burette et al., 1999); before the immunohistochemical incubations, sections were treated at $37^{\circ} \mathrm{C}$ for $10 \mathrm{~min}$ with pepsin ( $2 \mathrm{U} / \mathrm{ml}$ in acetic acid, $0.5 \mathrm{M})$ (Sigma, St. Louis, MO).

To assess antibody specificity, we performed experiments on transfected cells; C1-ABP (Wyszynski et al., 1999) (S. DeSouza, L. Khatri, and E. B. Biff, unpublished observations) or GRIP expression plasmids were introduced into $\mathrm{HeLa}$ cells using Effectene lipofection reagents (Qiagen, Valencia, CA). At $48 \mathrm{hr}$ after transfection, cells were fixed for $10 \mathrm{~min}$ in $0.5 \%$ depolymerized paraformaldehyde in PB or for $2 \mathrm{hr}$ in $4 \%$ paraformaldehyde in $\mathrm{PB}$.

$A B P$ immunostaining. For immunoperoxidase, free-floating sections fixed with $4 \%$ paraformaldehyde were treated for 30 min with $3 \% \mathrm{H}_{2} \mathrm{O}_{2}$ in PBS, pH 7.4 (to quench endogenous peroxidase activity), and then preincubated in $10 \%$ normal donkey serum (to block secondary antibody binding sites). Sections were incubated in primary antibody to ABP (1:1000 dilution) overnight on a shaker at room temperature. Sections were then incubated for $3 \mathrm{hr}$ in biotinylated anti-rabbit antibody (1:200) (Vector Laboratories, Burlingame, CA) and for $1 \mathrm{hr}$ in ExtrAvidinperoxidase complex (1:5000) (Sigma); peroxidase was histochemically visualized with diaminobenzidine. Processed sections were mounted on gelatin-coated slides and coverslipped with DPX mountant (BDH Chemicals, Poole, UK).

For immunofluorescence, tyramide signal amplification (TSA) permitted the use of primary antibody at dilutions of 1:10,000-1:20,000 (for tissue fixed with 4\% paraformaldehyde), and 1:40,000-1:100,000 (for tissue fixed with $0.5 \%$ paraformaldehyde). After overnight incubation in primary antibody and repeated washes in PBS containing $0.1 \%$ Tween 20 (PBS-T), the sections were reacted for $2 \mathrm{hr}$ at room temperature with biotinylated secondary antibody (1:200 in PBS) (Jackson ImmunoResearch, West Grove, PA). Biotin was localized by TSA (Renaissance direct) (DuPont NEN, Wilmington, DE); tissue was incubated in PBS containing $0.5 \%$ blocking reagent for $30 \mathrm{~min}$, then incubated in streptavidin-HRP (diluted 1:100 in PBS) for an additional $30 \mathrm{~min}$. Three washes with PBS-T were followed by a 5-7 min application of tyramide conjugated to FITC or $\mathrm{Cy} 3$, diluted 1:50 in amplification diluent, and then washed with PBS-T.

To obtain a better appreciation of the relationship between cellular morphology and immunostaining, we used the membrane tracer DiA (Molecular Probes, Eugene, OR), which labels even the finest neuronal

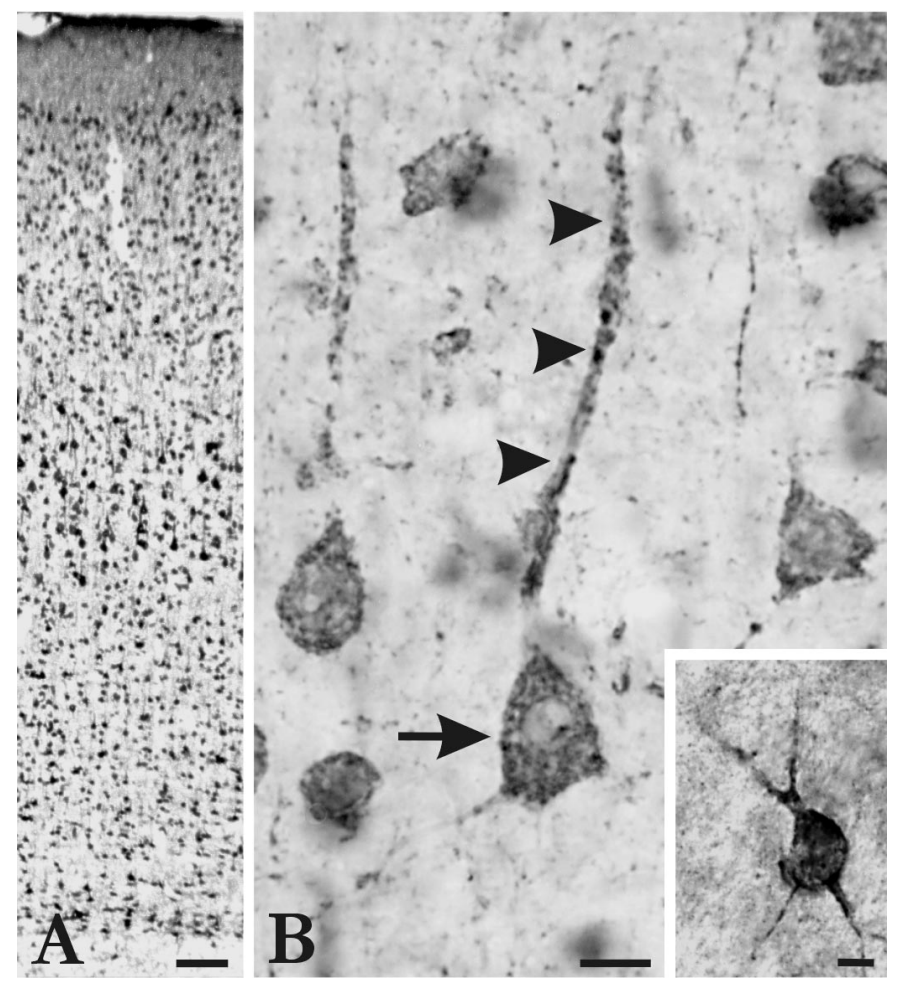

Figure 1. Immunostaining for $\mathrm{ABP}$ in cerebral cortex. $A$, Immunoperoxidase staining in $40-\mu \mathrm{m}$-thick tissue section, fixed with $4 \%$ paraformaldehyde. ABP immunoreactivity was found in neurons throughout all layers of cortex. B, Detail from layer V. Arrow points to a pyramidal neuron; staining is organized into patches in the soma and proximal part of the apical dendrite (arrowheads). Inset shows a nonpyramidal neuron with a characteristic $\mathrm{ABP}$-intense pattern of staining concentrated at the edge of the cell. Scale bars: $A, 100 \mu \mathrm{m} ; B, 20 \mu \mathrm{m}$; inset, $10 \mu \mathrm{m}$.

processes. DiA crystals were applied with a micropipette directly to some of the immunostained sections, which were then stored at $4^{\circ} \mathrm{C}$ for $24-72$ hr. Sections were mounted on gelatin-coated slides and either air-dried and cleared with xylene before being coverslipped with Fluoromount (Gurr BDH, Toronto, Canada) or directly coverslipped with Vectashield mounting medium (Vector Laboratories).

Multiple labeling. The first primary antibody was amplified with TSA, whereas the second and third primary antibodies were revealed with conventional fluorescent staining (Hunyady et al., 1996; Shindler and Roth, 1996). Sections were immunoprocessed for ABP as described above. After the application of the dye-conjugated tyramide and preincubation in $1 \%$ BSA in PBS-T, the second primary antibodies (either GRIP or GluR2/3 antibody, at dilutions of 1:1,000-1:2,000 for material fixed with $4 \%$ paraformaldehyde and 1:5,000-1:10,000, for material fixed with $0.5 \%$ paraformaldehyde) were applied overnight. Immunoreactivity was visualized with donkey anti-rabbit conjugated to rhodamine (Jackson ImmunoResearch). Sections labeled for ABP and GluR2/3 were then processed for synaptophysin (clone SVP-38, 1:1,000) (Sigma) or DiA labeling. Sections labeled for ABP and GRIP were then processed for synaptophysin, GluR2/3 (goat antibody Sc-7610) (Santa Cruz Biotechnology, Santa Cruz, CA), or DiA.

For optimal $z$-axis resolution, semithin plastic sections were prepared after immunofluorescence processing. Sections were then dehydrated and flat-embedded in Lowicryl HM20 (Polysciences, Warrington, PA). After polymerization, $1 \mu \mathrm{m}$ sections were cut, heat-mounted on slides, and coverslipped with Fluoromount.

To control for possible cross-reaction between the first primary antibody and the second secondary antibody (both raised in rabbit), the tissue was processed as described above, except that the second primary antibody was omitted. These sections were uniformly negative for the staining of the secondary antibodies. Moreover, we obtained consistent experimental results when the order of the first two primary antibodies was reversed.

Microscopy and data analysis. Sections were examined with a Leitz 

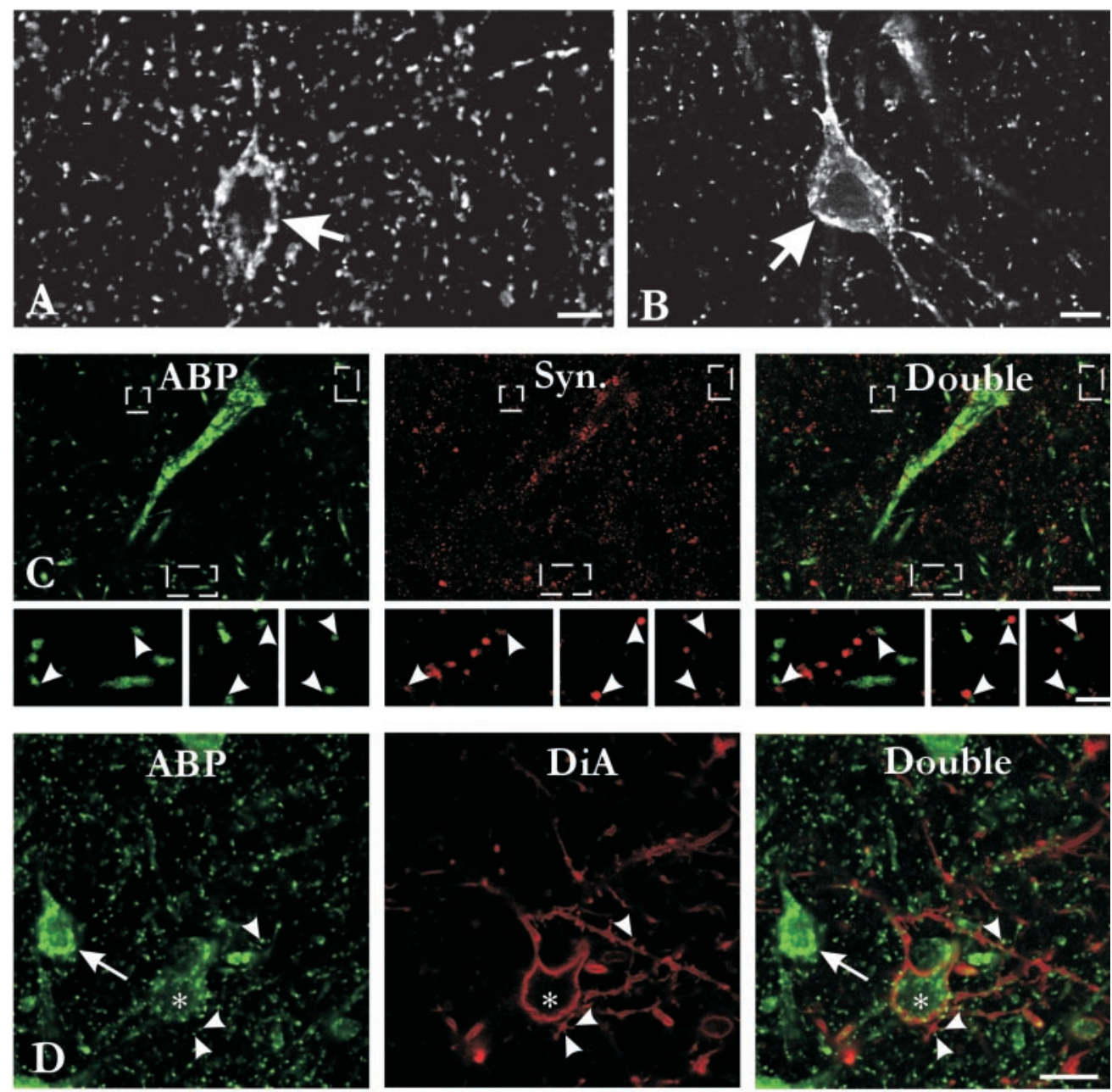

Figure 2. ABP immunofluorescent staining after fixation with $0.5 \%$ paraformaldehyde. $A, B$, Intense patchy staining was observed in somata (arrows) and dendrites. $C$, Numerous immunoreactive puncta were also present in the neuropil. These puncta often showed juxtaposition or partial overlap with synaptophysin (Syn.)-positive terminals (arrowheads on insets corresponding to the 3 boxes in main panel) and were thus likely to correspond to synapses. $D, E$, To elucidate the relationship of these puncta to cell processes, we performed double labeling with the
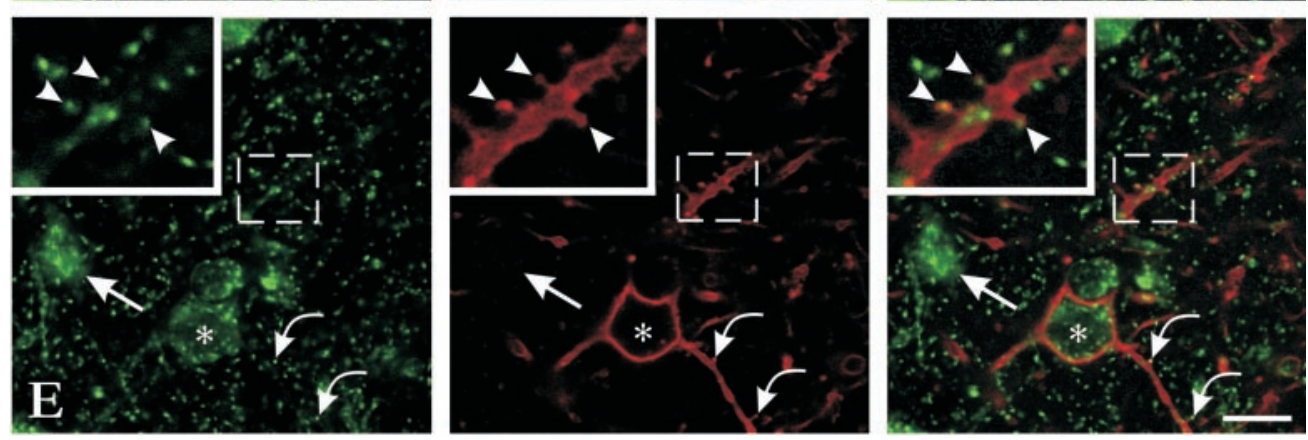
membrane tracer DiA (in $z$-axis stack, as illustrated by representative optical sections). Dense cellular staining of a small pyramidal neuron $(D, E$, arrows) contrasts with weaker $\mathrm{ABP}$ immunostaining in a nonpyramidal neuron (asterisk). DiA staining reveals that $\mathrm{ABP}$ concentrates at spines; most of these are likely to be on dendritic branches originating from pyramidal neurons $(D$, arrowheads; $E$, insets, arrowheads), but a few are on sparsely spiny dendrites of nonpyramidal neurons ( $E$, curved arrows). Scale bars: $A, B, 10$ $\mu \mathrm{m} ; C, 20 \mu \mathrm{m}$; insets, $5 \mu \mathrm{m} ; D, E, 25 \mu \mathrm{m}$.

DMR microscope under bright field, Nomarski, or epifluorescence illumination. Fluorescent images were acquired with a cooled chargecoupled device camera (Princeton Instruments, Trenton, NJ) coupled to a Macintosh computer. IP Lab software (Scanalytics, Fairfax, VA) was used for image acquisition and initial processing. For optimal resolution of thick sections, images were acquired with a Leica TCS confocal microscope. With optical sectioning, it was possible with this instrument to assess continuity of processes through the thickness of a section.

Quantification of puncta was performed on confocal images from randomly selected cortical fields. Images were acquired with a $100 \times$ oil objective with a pinhole diameter of 1.3 optical units (OUs) and $1 \times$ magnification. Quantification of immunopositive spines identified by DiA was performed on $25 \mu \mathrm{m}$ confocal $z$-axis stacks, acquired with a $63 \times$ oil objective with a pinhole diameter of $1.3 \mathrm{OU}$ and $1 \times$ magnification.

We also used the confocal instrument as a photometer to assess immunofluorescence staining of cells quantitatively. Randomly selected cortical fields were displayed at high magnification on the Leica TCS host computer screen. Neurons well defined by either ABP or GRIP immunocytochemistry were outlined, and mean pixel values of the outlined region were computed. Normalized immunofluorescence was computed as [(mean pixel value of cell) - (mean pixel value of background)]/ [(mean pixel value of brightest cell measured on section) - (mean pixel value of background)], yielding a dimensionless photometric value between 0 (immunofluorescence at background level) and 1 (the brightest cell measured). We used Corel Draw v.9 (Corel, Ontario, Canada) to sharpen images, adjust brightness and contrast level, and compose final plates.

\section{RESULTS}

\section{Distribution of ABP}

Most neurons in cerebral cortex were immunopositive for ABP. Both weakly and strongly stained neurons were present in all cortical layers (Fig. 1 $A$ ). Some of these could be identified as pyramidal neurons (Fig. $1 B$ ); others were nonpyramidal cells of various shapes and sizes. The degree of staining varied according to cell type: pyramidal neurons were generally more intensely 
A

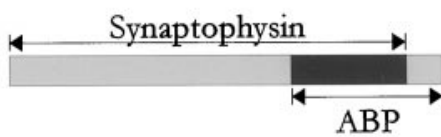

puncta

B

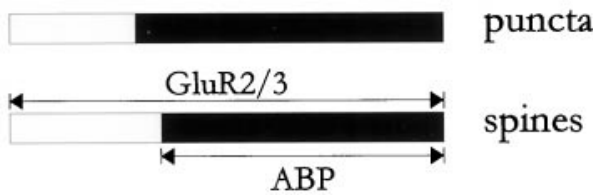

C

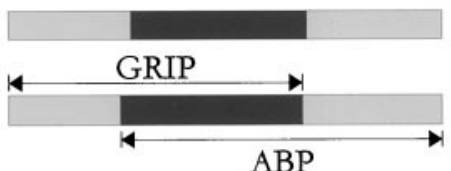

puncta

spines

Figure 3. Chart summarizing the quantitative data on synaptic localization of ABP and its colocalization with GluR2/3 and GRIP. $A$, A close relationship between ABP puncta and synaptophysin was observed in the neuropil; $76 \%$ of ABP puncta showed juxtaposition or partial overlap with a synaptophysin-positive punctum. $B$, Seventy-one percent of GluR2/3positive puncta were likely to be synaptic (as defined by synaptophysin), and $65 \%$ of GluR2/3-positive spines (identified by DiA) were immunopositive for ABP. $C$, Of puncta likely to represent synapses (as defined by synaptophysin) that were immunopositive for either ABP or GRIP, $40 \%$ colocalized ABP and GRIP, $31 \%$ were selectively positive for ABP, and $28 \%$ were selectively positive for GRIP. Results were similar for dendritic spines; of the spines immunopositive for either ABP or GRIP, $42 \%$ were stained for both ABP and GRIP, $32 \%$ for ABP alone, and $26 \%$ for GRIP alone.

stained for ABP than were nonpyramidal neurons. A few nonpyramidal neurons $(<1 \%$ of all labeled neurons) were particularly intensely immunopositive, with a characteristic pattern of staining mainly restricted to the edge of the cell, at or adjacent to the plasma membrane (Fig. 1B, inset). Although scattered through the cortex, these neurons were most frequently encountered in layers I-III. Except for these "ABP-intense" neurons, staining was typically patchy within proximal dendrites and somatic cytoplasm, excluding the nucleus. Immunopositive puncta were observed throughout the neuropil, particularly in layers I and II. We interpret these puncta as dendritic branches cut in cross-section, rather than immunoreactive synapses, because no clear association with the presynaptic marker synaptophysin could be detected in double-labeling experiments (data not shown).

The apparent lack of synaptic staining was unexpected, considering published evidence from postembedding immunoelectron microscopy (Srivastava et al., 1998). We reasoned that if ABP acts to anchor GluR2, it may be complexed with other proteins within the postsynaptic density, thus limiting antibody access. To explore whether there may be an occult synaptic pool of ABP only poorly detected with standard preembedding methods, we performed immunocytochemistry on sections pretreated with pepsin and on tissue only weakly fixed with paraformaldehyde. With both techniques, staining of puncta in the neuropil was considerably enhanced. Unfortunately, proteolytic treatment caused substantial damage to tissue sections and impaired cytoplasmic staining. The most satisfactory results were obtained from sections weakly fixed by $0.5 \%$ paraformaldehyde (Fig. 2). This procedure markedly increased the detection of antigen while suppressing background and thus provided optimal visualization of the cellular and subcellular distribution of ABP. In this material, intense patchy
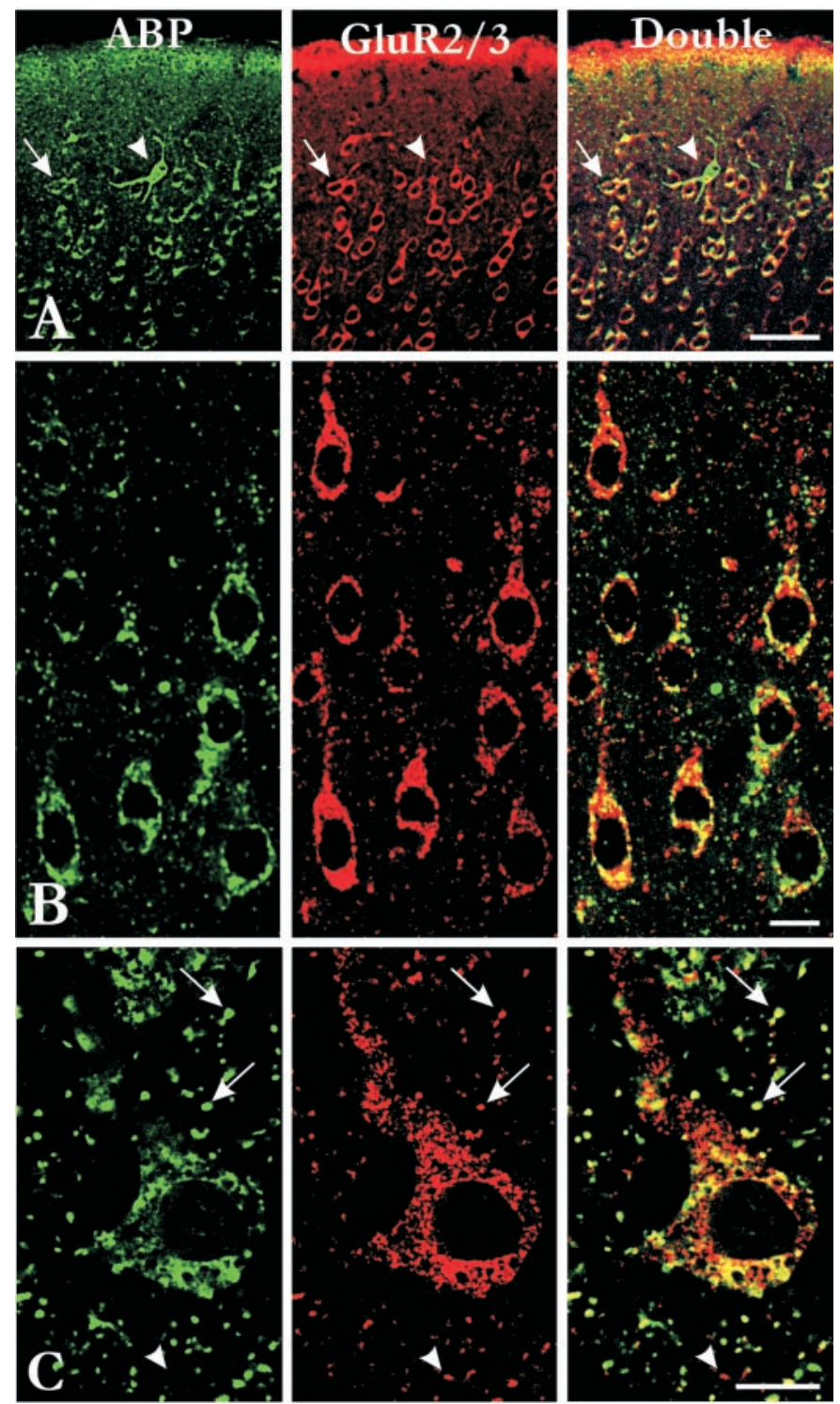

Figure 4. Colocalization of ABP with GluR2/3. $A, B$, Double labeling for ABP and GluR2/3 in layers I-II $(A)$ and III $(B)$, after fixation with $4 \%$ paraformaldehyde. Most GluR2/3-positive cells contained ABP immunoreactivity $(A$,arrows), with the exception of scattered ABP-intense neurons (arrowheads). In the neuropil, GluR2/3 puncta were seldom associated with $\mathrm{ABP}$ puncta after strong fixation. $C$, Double labeling for ABP and GluR2/3 in a large pyramidal neuron of layer $\mathrm{V}$, after fixation with $0.5 \%$ paraformaldehyde. In somata and proximal dendrites, ABP patches were sometimes associated with GluR2/3 patches, but many GluR2/3 patches were distinct from ABP patches. In contrast with the labeling after fixation with $4 \%$ paraformaldehyde, ABP-immunopositive puncta in the neuropil usually also stained for GluR2/3 (arrows), although some GluR2/3-positive puncta did not stain for ABP (arrowheads). Micrographs are from a $30 \mu \mathrm{m}$ frozen section $(A)$, a $1-\mu \mathrm{m}$-thick plastic section $(B)$, and a $50 \mu \mathrm{m}$ frozen section $(C)$. Scale bars: $A, 50 \mu \mathrm{m} ; B, C, 20 \mu \mathrm{m}$.

staining was observed in somata and proximal dendrites and in puncta throughout the neuropil (Fig. 2A,B). Double-labeling experiments showed a close relationship between many of these puncta and synaptophysin (Fig. $2 C$ ), indicating that weak fixation by $0.5 \%$ paraformaldehyde allowed the detection of the synaptic pool of ABP. Of 873 synaptophysin-positive puncta, 281 (32\%) were associated with an ABP-positive punctum. Conversely, of 

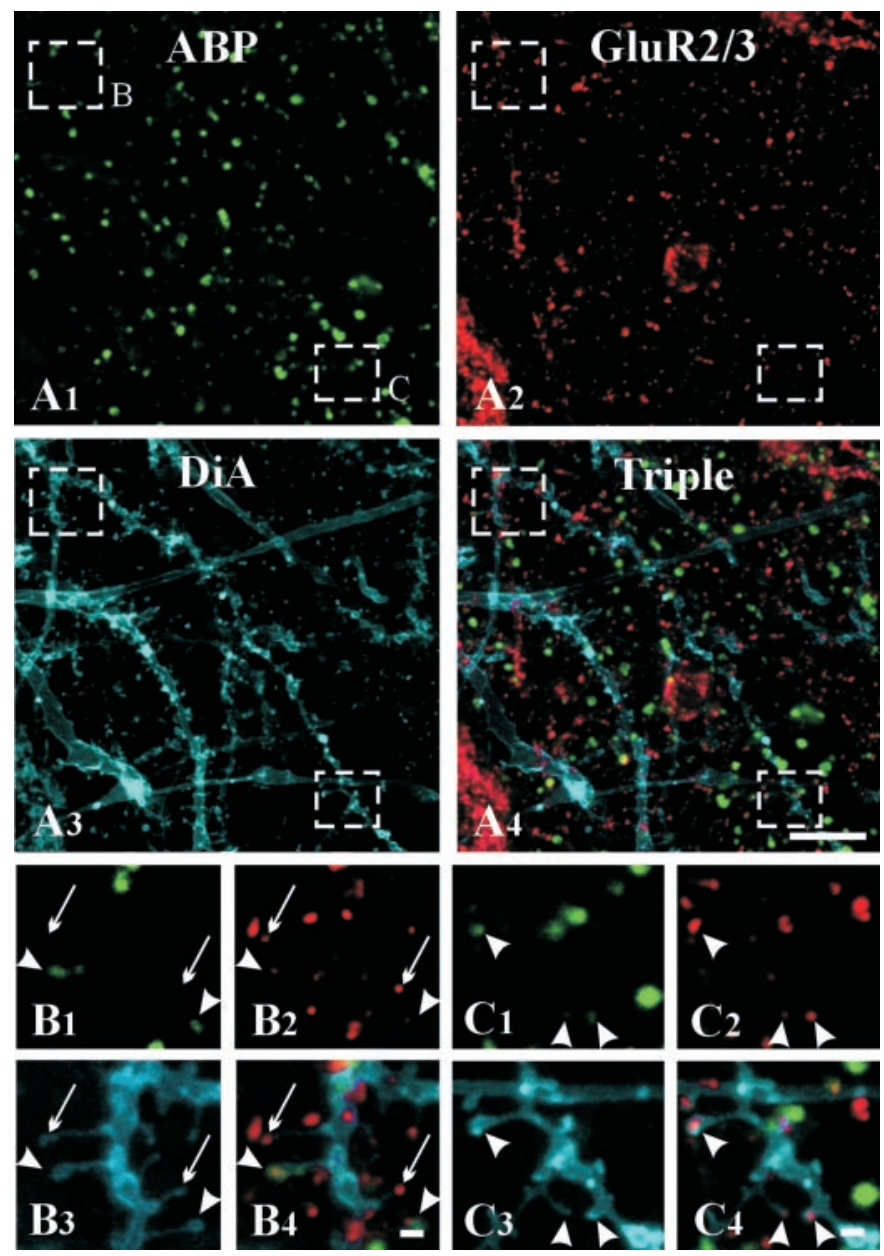

Figure 5. Partial colocalization of ABP with GluR2/3 in spines. DiA staining $(A 3)$ reveals spiny dendrites (boxed regions are shown at higher magnification in $B, C)$. Many of the spines are immunopositive for both ABP and GluR2/3 (arrowheads), whereas others are positive for only GluR2/3 (arrows). Scale bars: $A, 10 \mu \mathrm{m} ; B, C, 2 \mu \mathrm{m}$.

370 ABP-positive puncta, 281 (76\%) showed juxtaposition or partial overlap with a synaptophysin-positive punctum (Fig. $3 A$ ), suggesting that most immunocytochemically identified ABP puncta were synaptic. This synaptic localization was confirmed by double labeling with the membrane tracer DiA, which showed that many dendritic spines contain ABP (Fig. 2D,E).

\section{Colocalization of ABP with AMPA receptor subunits}

Previous studies have shown that ABP interacts with both GluR2 and GluR3 in vitro. If it also binds these subunits in vivo, one would expect ABP immunostaining in neurons immunopositive for GluR2/3. We therefore performed double labeling for ABP and AMPA receptors using antibodies that recognized both GluR2 and GluR3 subunits. Patterns of immunostaining for GluR2/3 were similar to previous reports (Petralia and Wenthold, 1992). In the cerebral cortex, GluR $2 / 3$ antibodies stained somata and dendrites of a large population of pyramidal cells and also stained a substantial number of nonpyramidal cells. Double labeling showed that many GluR2/3-immunopositive neurons also expressed high levels of ABP, but a substantial fraction expressed $\mathrm{ABP}$ at low or undetectable levels. In contrast, the vast majority of ABP-positive cells also stained for GluR2/3, with the exception of the scattered ABP-intense neurons, which generally lacked GluR2/3 (Fig. 4A,B).

In somata, patches of ABP immunoreactivity occasionally colocalized with GluR2/3 patches, but more typically, these patches seemed independently organized (Fig. 4C). Considering the dense labeling for both antigens, the overall impression was of a lack of association. In contrast, punctate colocalization of ABP and GluR2/3 was often observed in the neuropil (Fig. 4B,C), although some GluR2/3 puncta were immunonegative for ABP. By combining immunolabeling with DiA, we could demonstrate that although many dendritic spines were immunopositive for both markers, many others were positive for GluR2/3 but not ABP (Fig. 5).

To assess the relative frequencies of synapses of these two types, we performed triple immunolabeling for GluR2/3, ABP, and synaptophysin (data not shown). Of 522 GluR2/3-positive puncta likely to be synaptic (as defined by synaptophysin), 373 (71\%) were also immunopositive for ABP (Fig. 3B). Using a second approach to identify putative synaptic labeling, we found that of 54 GluR2/3-positive spines (identified by DiA), 35 (65\%) were also immunopositive for ABP (Fig. $3 B$ ). That a negative spine could be adjacent to an immunopositive spine (Fig. 5B) documents a high level of chemical heterogeneity, even within a single dendritic twig.
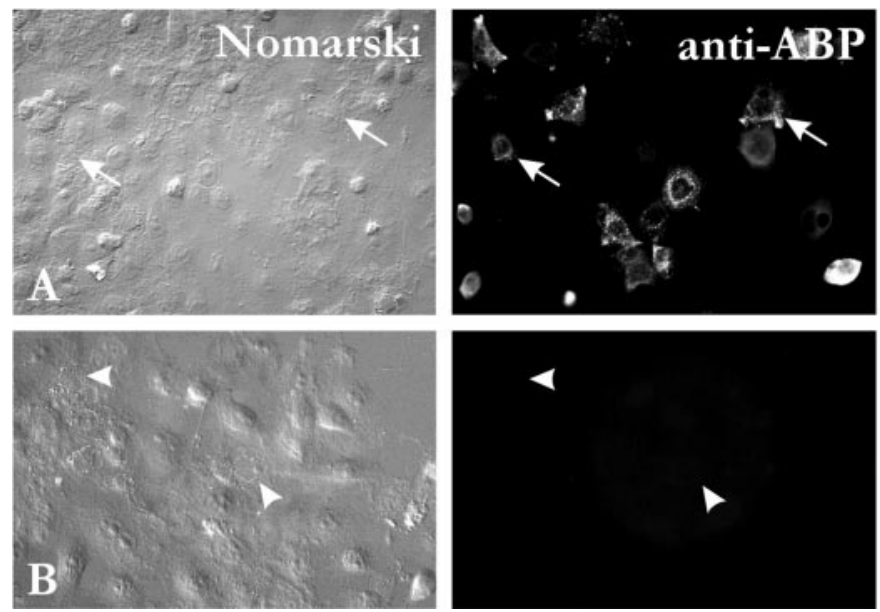

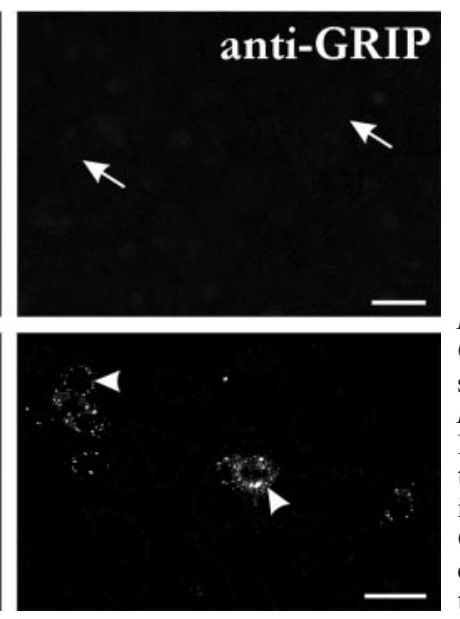

Figure 6. Specificity of the $\mathrm{ABP}$ and GRIP antibodies used in the present study. $A$, Immunofluorescence for ABP. $B$, Immunofluorescence for GRIP (1756/ B. O.). ABP was detected in HeLa cells transfected with $\mathrm{ABP}(A$, arrows), but not in GRIP-transfected cells. Likewise, GRIP was detected in GRIP-transfected cells ( $B$, arrowheads), but not in ABPtransfected cells. Scale bars, $50 \mu \mathrm{m}$. 

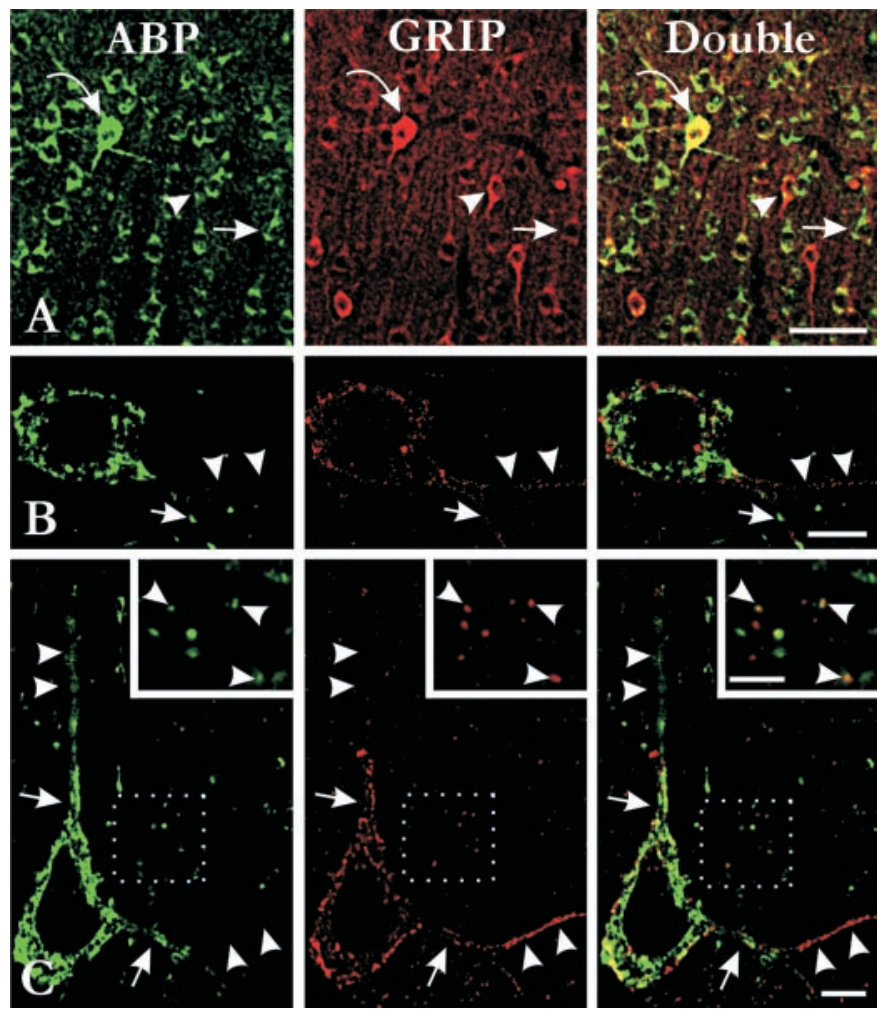

Figure 7. Partial immunocolocalization of ABP with GRIP. A, Double labeling for ABP and GRIP in the cerebral cortex; tissue fixed with $4 \%$ paraformaldehyde. Most ABP-positive cells contained GRIP immunoreactivity, and vice versa. However, cells strongly immunopositive for ABP (small arrows) were weakly stained for GRIP, whereas cells strongly immunopositive for GRIP (arrowheads) were weakly stained for ABP. Scattered ABPintense neurons were strongly positive for both proteins (curved arrows). $B, C$, Double labeling for ABP and GRIP after fixation with $0.5 \%$ paraformaldehyde. Intrasomatic patches of ABP immunoreactivity were generally distinct from patches of GRIP. A differential distribution of ABP and GRIP was often observed within the dendritic arborization. $B$, A proximal dendrite and one of its branches (arrows) were stained for both proteins, but the other branch was stained for GRIP alone (arrowheads). $C$, The proximal apical dendrite was stained for both proteins (arrows), but more distal regions were stained for ABP alone (arrowheads); likewise, the proximal basal dendrite was stained for both proteins (arrows), but distal branches were stained for GRIP alone (arrowheads). Insets show that many puncta in the neuropil colocalized both ABP and GRIP (arrowheads), but a number of puncta were selectively positive for either GRIP or ABP. Scale bars: $A, 50 \mu \mathrm{m} ; B, C, 10$ $\mu \mathrm{m}$; inset, $5 \mu \mathrm{m}$.

\section{Colocalization of ABP and GRIP}

That ABP and GluR2/3 exhibit only partial colocalization suggests that a sizable fraction of GluR2/3-positive synapses may lack ABP. This led us to explore the relationship between ABP and GRIP, another putative GluR2/3 scaffold. Because ABP (outside its L2 region) is homologous to GRIP, there is the theoretical possibility of cross-reaction between GRIP antisera and ABP. To assess this possibility, we performed immunofluorescence on transfected cells. ABP immunoreactivity was detected in ABPtransfected cells, but not in GRIP-transfected cells (Fig. 6A). Likewise, immunoreactivity for GRIP (antibody 1756/B.O.) was detected in GRIP-transfected cells, but not in ABP-transfected cells (Fig. 6B). These data suggest that there was no significant cross-reaction between ABP and GRIP antibodies, at least at the concentration used in this work. To verify the reliability of our results, the observations reported below were qualitatively confirmed using a second GRIP antibody, prepared against a peptide

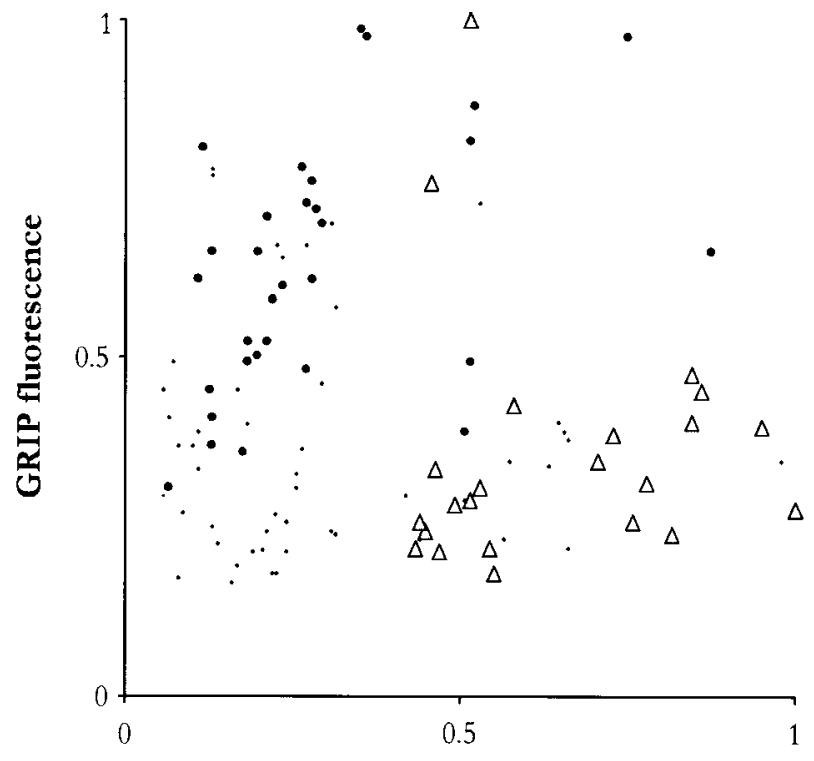

ABP fluorescence

Figure 8. Relative intensities of ABP and GRIP immunofluorescence in cerebral cortex. Intensity of somatic labeling was measured in 103 neurons spanning all cortical layers from a representative double-stained section. Confocal images were captured, and mean photometric intensities of red and green channels were computed for each that was well defined in either channel; data were normalized from 0 (tissue background) to 1 (brightest cell measured) for each channel. Open triangles represent morphologically identified pyramidal cells, filled circles represent nonpyramidal neurons, and dots represent neurons that could not be clearly identified. The two types of neurons form distinct clusters; pyramidal neurons generally stained strongly for ABP and not for GRIP, whereas nonpyramidal neurons stained strongly for GRIP and not for ABP. Outlying cells, top right, were exceptionally large. A cluster of cells near the origin was stained too weakly to permit clear identification.

sequence not shared by ABP (deSouza and Ziff, unpublished observations).

Cellular staining for GRIP was consistent with previous reports (Wyszynski et al., 1998; Burette et al., 1999; Dong et al., 1999): GRIP stained dendrites and somata of nonpyramidal neurons scattered throughout cortex, and pyramidal cells were only weakly immunopositive. Most GRIP-positive cells also contained $\mathrm{ABP}$, and vice versa (Fig. 7A). Cells strongly immunopositive for ABP (mainly pyramidal neurons) were weakly stained for GRIP, whereas cells strongly immunopositive for GRIP (a subset of nonpyramidal neurons) were weakly stained for ABP; in contrast, the scattered ABP-intense neurons were usually strongly positive for both proteins (Fig. 7A). Photometry of cells from random high-power fields (see Materials and Methods) confirmed this impression (Fig. 8): immunofluorescence for ABP was significantly ( $p<0.001$; two-sided $t$ test) stronger for pyramidal cells (mean normalized brightness, $0.640 \pm 0.040 ; n=23$ ) than for nonpyramidal neurons $(0.286 \pm 0.033 ; n=32)$, whereas immunofluorescence for GRIP was significantly $(p<.001)$ weaker for pyramidal cells $(0.360 \pm 0.039)$ than for other neurons $(0.629 \pm$ $0.033)$.

Subcellular colocalization was not the rule within the cell body; intrasomatic patches of ABP immunoreactivity were generally distinct from patches stained for GRIP (Fig. 7B,C). In some cases, ABP and GRIP were differentially distributed within a single dendritic arborization: the proximal dendrite was stained for both proteins, but secondary dendrites were stained for either ABP or GRIP alone (Fig. $7 B, C$ ). Beyond suggesting differential 

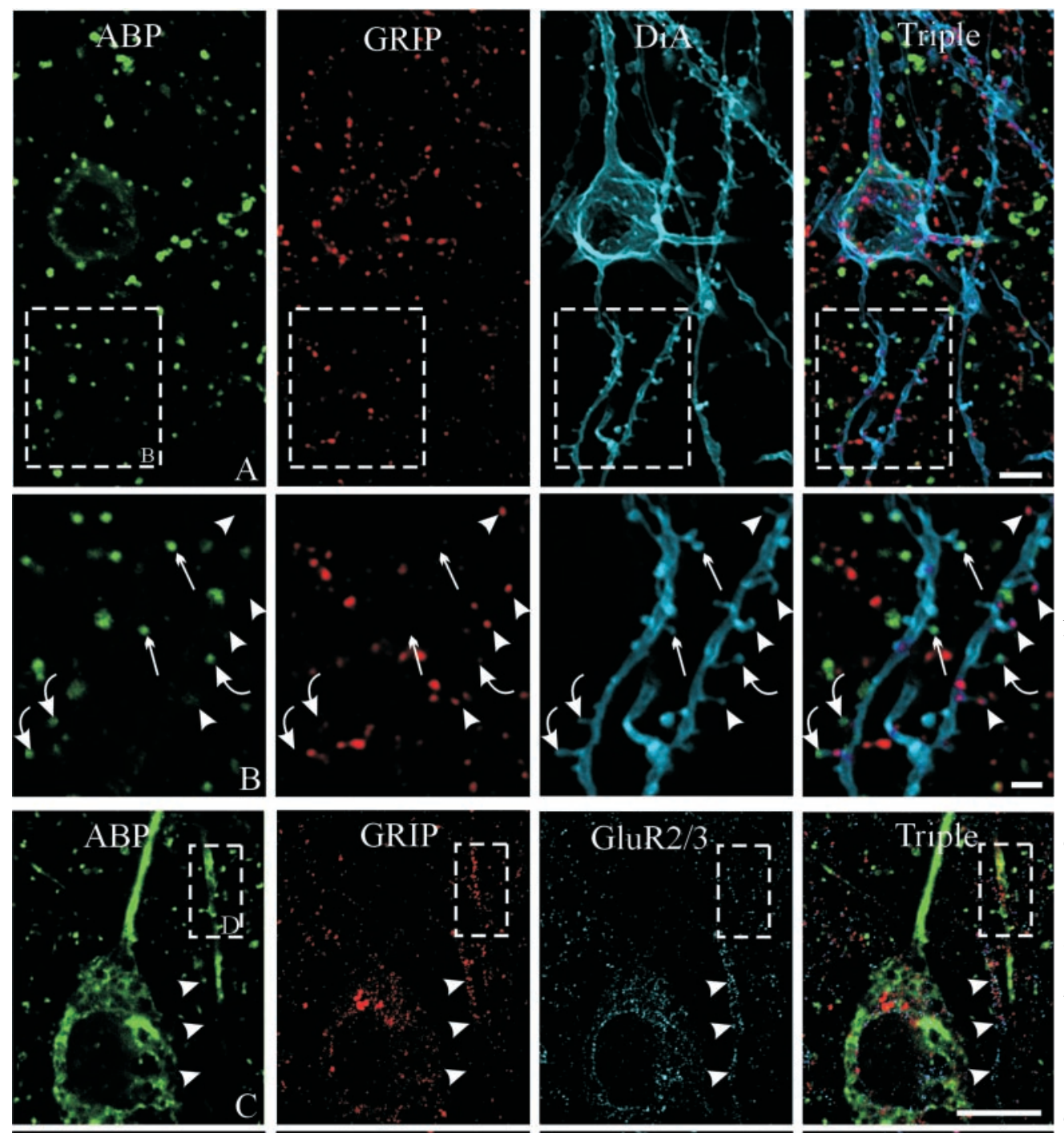

Figure 9. Partial colocalization of ABP with GRIP in GluR2/3-immunoreactive spines. $A, B$, DiA staining reveals a pyramidal neuron stained for both $\mathrm{ABP}$ and GRIP. $B$, Higher-magnification view of spiny dendrites shown in $A$. Some spines are immunopositive for both $\mathrm{ABP}$ and GRIP (curved arrows), whereas others are immunopositive for ABP alone (straight arrows) or GRIP alone (arrowheads). Note that spines with two different chemical profiles originate from the same dendrite. $C, D$, Triple immunostaining for ABP, GRIP, and GluR2/3. C, Two vertically directed dendrites are visible on the right. One is immunopositive for GRIP and GluR2/3 (arrowheads) but shows virtually no staining for ABP. The other (boxed areas) is immunostained for all three antigens. $D$, Enlargement of boxed areas shows GluR2/3-positive spines im-
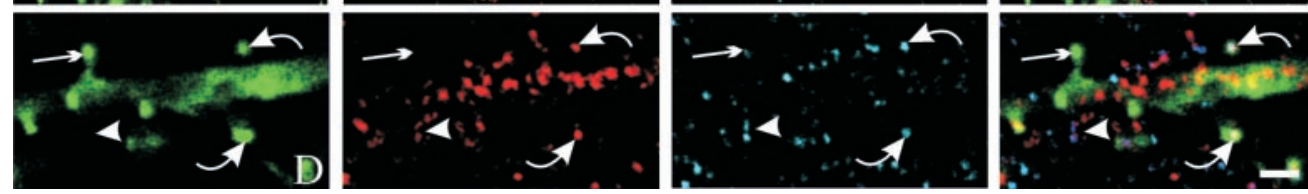
munopositive for both ABP and GRIP (curved arrows), for ABP only (straight arrows), and GRIP only (arrowheads), all associated with the same dendrite. Scale bars: $A, 10 \mu \mathrm{m} ; B, 50 \mu \mathrm{m} ; C, 20 \mu \mathrm{m}$; $D, 3 \mu \mathrm{m}$.

transport and/or targeting of the two proteins, these data provide further evidence that the two antibodies recognized different antigens.

Of puncta immunopositive for either ABP or GRIP, we identified 683 that were likely to represent synapses because they either overlapped or were adjacent to synaptophysin-positive puncta. Of these, 274 (40\%) colocalized ABP and GRIP, 216 (31\%) were selectively positive for ABP, and 193 (28\%) were selectively positive for GRIP (Fig. 3C). Consistent with this finding, of 53 spines immunopositive for either ABP or GRIP (as revealed by DiA staining), $22(42 \%)$ were stained for both ABP and GRIP, $17(32 \%)$ for ABP alone, and 14 (26\%) for GRIP alone (Figs. $3 C, 8 A, B$ ). The three types of immunostaining (i.e., ABP only, GRIP only, and colocalization of both) could be observed on adjacent spines of the same dendrite (Fig. 9A,B). These results, together with the quantitative data on ABP staining of GluR2/3-positive synapses described above, are consistent with the possibility that all GluR2/3-positive synapses contain either ABP or GRIP or both. We then examined spines triplelabeled with GluR2/3, ABP, and GRIP. This combination of labeling required histochemical procedures that yielded suboptimal staining, but we were able to confirm colocalization of GluR2/3-positive spines with both of these scaffolding proteins in different combinations (Fig. 9C,D).

\section{DISCUSSION}

Using multiple-label immunocytochemistry, we have characterized the cellular and subcellular expression of ABP and its relationships with GluR2/3 and GRIP. Immunostaining for ABP in the cerebral cortex was strong in pyramidal neurons and weaker in nonpyramidal neurons. All three antigens were also found at synapses, but only after proteolytic pretreatment or in weakly fixed tissue, suggesting that ABP/GRIP may be so tightly complexed with AMPA receptors and other proteins at the synapse that antibody access is severely restricted (Ye et al., 2000). 


\section{$A B P$ associates with GluR2/3 in vivo}

In vitro evidence suggests a role for ABP in anchoring AMPA receptors. Yeast two-hybrid assays show that ABP can bind via its PDZ domains 3, 5, and 6 to the C termini of GluR2 and GluR3; furthermore, ABP copurifies with GluR2 in the PSD fraction of brain homogenate (Srivastava et al., 1998). The multiple labeling experiments reported here are consistent with the idea that ABP and GluR2/3 interact in vivo. Although ABP is expressed almost exclusively in GluR2/3-containing neurons, high-resolution images show little subcellular colocalization of the two antigens in somata and proximal dendrites. In contrast, colocalization in the neuropil is prominent, and a large fraction of this colocalization is likely to be synaptic, as shown by its association with dendritic spines and with synaptophysin. Together, these observations suggest that $\mathrm{ABP}$ is not involved in cytoplasmic receptor trafficking, but may play an important role at or near the synapse. The role of ABP in the small group of ABP-intense, GluR2/3-negative cells remains unclear. For their morphology, location, and intense staining for GRIP, we suspect that these are likely to represent a specialized population of GluR1-rich GABAergic interneurons (Burette et al., 1999), suggesting that ABP and GRIP play some scaffolding role unrelated to GluR2/3 in these neurons.

\section{ABP and GRIP are differentially expressed}

Notwithstanding extensive cellular colocalization of GRIP and ABP, the relative intensity of labeling for the two antigens varied: cells strongly immunopositive for ABP (mainly pyramidal neurons) were typically weakly stained for GRIP, whereas cells strongly immunopositive for GRIP (mainly nonpyramidal neurons) were weakly stained for ABP. Sparsely spiny or aspiny dendrites likely to originate from nonpyramidal neurons (and previously shown to express GRIP at high levels) (Burette et al., 1999) typically contained markedly less ABP than did spiny dendrites.

We used both dendritic spines and synaptophysin as light microscopic proxies to investigate patterns of synaptic colocalization. Both proxies are dominated by pyramidal neurons: spines originate overwhelmingly from pyramidal neurons, but even a random sample of asymmetric synapses (as revealed by synaptophysin) will be dominated by axospinous synapses, considering the relative infrequency of excitatory synapses on dendritic shafts in neocortex (Feldman, 1984). Keeping this caveat in mind, our data suggest that although many of these synapses contained both GRIP and ABP, the majority were positive for only one of the proteins. Thus, ABP and GRIP must be independently regulated; that the three types of immunostaining (ABP only, GRIP only, and colocalization) could be observed on adjacent spines of the same dendrite shows that this regulation is very tight indeed. Notwithstanding the differential distribution of the two antigens, we conclude from the present results that most, or perhaps all, GluR2/3-containing synapses in cerebral cortex include either ABP or GRIP.

\section{Possible functional implications}

Synaptic AMPA receptors appear to be more firmly anchored at GABAergic (nonpyramidal) cells than at pyramidal neurons (Allison et al., 1998). In the light of the present evidence, this suggests that AMPA receptors are less mobile at GRIP-enriched synapses than at ABP-enriched synapses. This raises the possibility that GRIP might bind GluR2/3 more firmly than does ABP; it would be interesting to test this hypothesis directly. Heterologous expression experiments suggest that ABP and GRIP may copolymerize to form large scaffolds within the PSD (Srivastava et al., 1998; Dong et al., 1999), leading to the more general hypothesis that the ratio of ABP/GRIP may control the mobility of GluR2/3 within the synapse. Because addition of new AMPA receptors to the synapse may underlie some forms of long-term potentiation (LTP) (for review, see Malinow, 1998; Malenka and Nicoll, 1999; Turrigiano, 2000), the limited available evidence suggesting more robust LTP in pyramidal than in nonpyramidal neurons (McMahon and Kauer, 1997; Laezza et al., 1999; McBain et al., 1999) is consistent with this hypothesis.

In addition to their role as receptor anchors, both GRIP and ABP are likely to act as a molecular scaffold, organizing intracellular second-messenger cascades. Notwithstanding their close homology (64-93\% in their six PDZ domains), it seems likely that the two proteins have different binding affinities for intracellular kinases and phosphatases that may be important in synaptic plasticity (Torres et al., 1998; Bhalla and Iyengar, 1999; Bruckner et al., 1999; Bloomfield and Ziff, 2000; Soderling and Derkach, 2000) (M. Wyszynski and M. Sheng, unpublished observations). Interestingly, the novel RasGEF GRASP-1 binds to the seventh PDZ domain of GRIP and its ABP homolog, ABP-L, but not to ABP (which lacks the seventh PDZ domain) (Ye et al., 2000), suggesting functional consequences of ABP splice variant regulation. The present research points to the need for further study of potential intracellular binding partners for these scaffolding molecules.

\section{REFERENCES}

Allison DW, Gelfand VI, Spector I, Craig AM (1998) Role of actin in anchoring postsynaptic receptors in cultured hippocampal neurons: differential attachment of NMDA versus AMPA receptors. J Neurosci 18:2423-2436.

Bekkers JM, Stevens CF (1989) NMDA and non-NMDA receptors are colocalized at individual excitatory synapses in cultured rat hippocampus. Nature 341:230-233.

Bhalla US, Iyengar R (1999) Emergent properties of networks of biological signaling pathways. Science 283:381-387.

Bloomfield S, Ziff EB (2000) Novel interactions with AMPA receptor binding protein. Soc Neurosci Abstr 26:527.

Bruckner K, Pablo Labrador J, Scheiffele P, Herb A, Seeburg PH, Klein R (1999) EphrinB ligands recruit GRIP family PDZ adaptor proteins into raft membrane microdomains. Neuron 22:511-524.

Burette A, Wyszynski M, Valtschanoff JG, Sheng M, Weinberg RJ (1999) Characterization of glutamate receptor interacting proteinimmunopositive neurons in cerebellum and cerebral cortex of the albino rat. J Comp Neurol 411:601-612.

Conti F, Weinberg RJ (1999) Shaping excitation at glutamatergic synapses. Trends Neurosci 22:451-458.

Craven SE, Bredt DS (1998) PDZ proteins organize synaptic signaling pathways. Cell 93:495-498.

Dong H, Zhang P, Song I, Petralia RS, Liao D, Huganir RL (1999) Characterization of the glutamate receptor-interacting proteins GRIP1 and GRIP2. J Neurosci 19:6930-6941.

Feldman LF (1984) Morphology of the neocortical pyramidal neuron. In: Cerebral cortex (Peters A, Jones EG, eds), pp 123-189. New York: Plenum.

He Y, Janssen WG, Morrison JH (1998) Synaptic coexistence of AMPA and NMDA receptors in the rat hippocampus: a postembedding immunogold study. J Neurosci Res 54:444-449.

Hunyady B, Krempels K, Harta G, Mezey E (1996) Immunohistochemical signal amplification by catalyzed reporter deposition and its application in double immunostaining. J Histochem Cytochem 44:1353-1362.

Kennedy MB (1997) The postsynaptic density at glutamatergic synapses. Trends Neurosci 20:264-268.

Kharazia VN, Weinberg RJ (1997) Tangential synaptic distribution of NMDA and AMPA receptors in rat neocortex. Neurosci Lett 238:41-44.

Kharazia VN, Phend KD, Rustioni A, Weinberg RJ (1996) EM colocalization of AMPA and NMDA receptor subunits at synapses in rat cerebral cortex. Neurosci Lett 210:37-40.

Kornau HC, Schenker LT, Kennedy MB, Seeburg PH (1995) Domain interaction between NMDA receptor subunits and the postsynaptic density protein PSD-95. Science 269:1737-1740. 
Laezza F, Doherty JJ, Dingledine R (1999) Long-term depression in hippocampal interneurons: joint requirement for pre- and postsynaptic events. Science 285:1411-1414.

Malenka RC, Nicoll RA (1999) Long-term potentiation: a decade of progress? Science 285:1870-1874.

Malinow R (1998) Silencing the controversy in LTP? Neuron 21:1226-1227.

McBain CJ, Freund TF, Mody I (1999) Glutamatergic synapses onto hippocampal interneurons: precision timing without lasting plasticity. Trends Neurosci 22:228-235.

McMahon LL, Kauer JA (1997) Hippocampal interneurons express a novel form of synaptic plasticity. Neuron 18:295-305.

Migaud M, Charlesworth P, Dempster M, Webster LC, Watabe AM, Makhinson M, He Y, Ramsay MF, Morris RG, Morrison JH, O'Dell TJ, Grant SG (1998) Enhanced long-term potentiation and impaired learning in mice with mutant postsynaptic density-95 protein. Nature 396:433-439.

Niethammer M, Kim E, Sheng M (1996) Interaction between the C terminus of NMDA receptor subunits and multiple members of the PSD-95 family of membrane-associated guanylate kinases. J Neurosci 16:2157-2163.

Nusser Z (2000) AMPA and NMDA receptors: similarities and differences in their synaptic distribution. Curr Opin Neurobiol 10:337-341.

O'Brien RJ, Lau LF, Huganir RL (1998) Molecular mechanisms of glutamate receptor clustering at excitatory synapses. Curr Opin Neurobiol 8:364-369.

Ottersen OP, Landsend AS (1997) Organization of glutamate receptors at the synapse. Eur J Neurosci 9:2219-2224.

Petralia RS, Wenthold RJ (1992) Light and electron immunocytochemical localization of AMPA-selective glutamate receptors in the rat brain. J Comp Neurol 318:329-354

Ponting CP, Phillips C, Davies KE, Blake DJ (1997) PDZ domains: targeting signalling molecules to sub-membranous sites. BioEssays 19:469-479.

Scott K, Zuker CS (1998) Assembly of the Drosophila phototransduction cascade into a signalling complex shapes elementary responses. Nature 395:805-808.

Sheng M, Wyszynski M (1997) Ion channel targeting in neurons. BioEssays 19:847-853.
Shindler KS, Roth KA (1996) Double immunofluorescent staining using two unconjugated primary antisera raised in the same species. J Histochem Cytochem 44:1331-1335.

Soderling TR, Derkach VA (2000) Postsynaptic protein phosphorylation and LTP. Trends Neurosci 23:75-80.

Somogyi P, Tamas G, Lujan R, Buhl EH (1998) Salient features of synaptic organisation in the cerebral cortex. Brain Res Brain Res Rev 26:113-135.

Srivastava S, Osten P, Vilim FS, Khatri L, Inman G, States B, Daly C, DeSouza S, Abagyan R, Valtschanoff JG, Weinberg RJ, Ziff EB (1998) Novel anchorage of GluR2/3 to the postsynaptic density by the AMPA receptor-binding protein ABP. Neuron 21:581-591.

Takumi Y, Ramirez-Leon V, Laake P, Rinvik E, Ottersen OP (1999) Different modes of expression of AMPA and NMDA receptors in hippocampal synapses. Nat Neurosci 2:618-624.

Torres R, Firestein BL, Dong H, Staudinger J, Olson EN, Huganir RL, Bredt DS, Gale NW, Yancopoulos GD (1998) PDZ proteins bind, cluster, and synaptically colocalize with Eph receptors and their ephrin ligands. Neuron 21:1453-1463.

Turrigiano GG (2000) AMPA receptors unbound: membrane cycling and synaptic plasticity. Neuron 26:5-8.

Watanabe M, Fukaya M, Sakimura K, Manabe T, Mishina M, Inoue Y (1998) Selective scarcity of NMDA receptor channel subunits in the stratum lucidum (mossy fibre-recipient layer) of the mouse hippocampal CA3 subfield. Eur J Neurosci 10:478-487.

Wyszynski M, Kim E, Yang FC, Sheng M (1998) Biochemical and immunocytochemical characterization of GRIP, a putative AMPA receptor anchoring protein, in rat brain. Neuropharmacology 37:1335-1344.

Wyszynski M, Valtschanoff JG, Naisbitt S, Dunah AW, Kim E, Standaert DG, Weinberg R, Sheng M (1999) Association of AMPA receptors with a subset of glutamate receptor-interacting protein in vivo. J Neurosci 19:6528-6537.

Ye B, Liao D, Zhang X, Zhang P, Dong H, Huganir RL (2000) GRASP-1: a neuronal RasGEF associated with the AMPA receptor/ GRIP complex. Neuron 26:603-617.

Ziff EB (1997) Enlightening the postsynaptic density. Neuron 19:11631174 . 